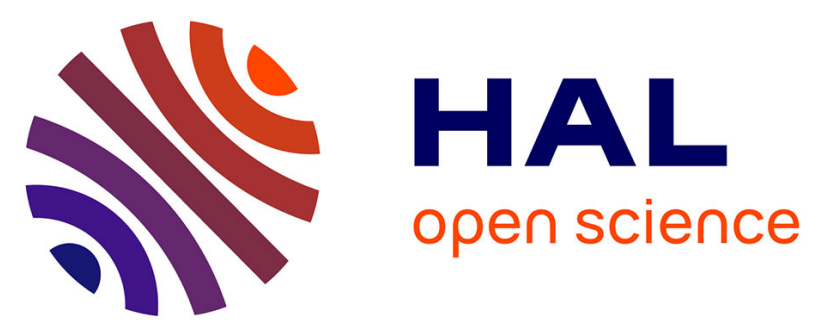

\title{
Etude de la flore microbienne du fromage espagnol" Manchego ". I. - SON EVOLUTION AU COURS DE LA FABRICATION ET DE L'AFFINAGE
}

\author{
Maria Roman Pinana, F. Jorganes, D. Gomez
}

\section{To cite this version:}

Maria Roman Pinana, F. Jorganes, D. Gomez. Etude de la flore microbienne du fromage espagnol " Manchego ". I. - SON EVOLUTION AU COURS DE LA FABRICATION ET DE L'AFFINAGE. Le Lait, 1975, 55 (547), pp.401-413. hal-00928696

\section{HAL Id: hal-00928696 https://hal.science/hal-00928696}

Submitted on 1 Jan 1975

HAL is a multi-disciplinary open access archive for the deposit and dissemination of scientific research documents, whether they are published or not. The documents may come from teaching and research institutions in France or abroad, or from public or private research centers.
L'archive ouverte pluridisciplinaire HAL, est destinée au dépôt et à la diffusion de documents scientifiques de niveau recherche, publiés ou non, émanant des établissements d'enseignement et de recherche français ou étrangers, des laboratoires publics ou privés. 


\title{
Etude de la flore microbienne du fromage espagnol \\ "Manchego"
}

\section{I. - SON EVOLUTION AU COURS DE LA FABRICATION ET DE L'AFFINAGE}

\author{
par \\ Maria ROMAN PINANA
}

(avec la collaboration technique de Mlles F. JORGANES et D. GÓMEZ)

Instituto de Productos Lácteos, C.S.I.C. Arganda del Rey, Madrid

\section{INTRODUCTION}

Par son volume de production (environ $3000000 \mathrm{~kg}$ par an) et son acceptation commerciale, le fromage "Manchego " est le plus important des fromages élaborés en Espagne [7]. Il est produit à partir du lait de brebis dans une région de climat continental, La Mancha, située dans le plateau central de la péninsule ibérique. Ce fromage, à pâte dure et de couleur blanchâtre, a un goût et un arôme caractéristiques.

Depuis ces dernières années, la production industrielle a supplanté la fabrication artisanale à partir de lait cru. Pour des raisons sanitaires et afin d'éliminer les germes pathogènes et autres microorganismes indésirables, d'autant plus prolifiques que la région de production est soumise à des températures élevées, les fromageries ont été obligées de pasteuriser le lait. L'inconvénient de cette opération est la destruction des bactéries lactiques indispensables à la fabrication du fromage " Manchego » typique. L'adjonction d'un levain de souches lactiques au lait pasteurisé devient donc une opération essentielle dans les fromageries. Les fromages produits dans ces conditions présentent souvent les meilleures caractéristiques de production artisanale d'autrefois mais l'homogénéité des produits n'est pas toujours assurée.

De nombreux travaux ont établi le rôle primordial joué par les micro-organismes et leurs enzymes dans le processus de maturation des fromages [5, 16]. Par ailleurs, l'ensemble des phénomènes biochimiques qui dominent ce processus détermine le développement 
de l'arôme et du goût caractéristiques du fromage. Malgré l'importance économique du " Manchego » aucune étude approfondie, concernant la nature de la flore microbienne dans la fabrication et la maturation de ce fromage, n'a été entreprise. Nous nous sommes donc proposés de suivre, au cours de la production industrielle, l'évolution de cette flore, et notamment, les variations du nombre des principaux groupes de micro-organismes qui la constituent, tant en surface qu'en profondeur. Ces études devraient permettre un meilleur contrôle du processus de fabrication du fromage « Manchego ».

\section{MATERIELS ET METHODES}

\section{Prélèvement des échantillons et technologie de fabrication}

Notre étude a porté sur deux séries de fromages fabriqués à partir de lait pasteurisé dans deux usines différentes (A et B), de la région de Madrid. Chaque série provient d'un même lot de fabrication. Dans chacune des fromageries ont été prélevés :

- des échantillons de lait de brebis avant et après pasteurisation,

- des échantillons de fromage aux différents stades de la fabrication et de l'affinage (tab. 1).

On prélève chaque fois sur un fromage différent, provenant d'une même fabrication, deux échantillons :

- un échantillon de surface, soit par grattage de chacune des faces (fromagerie B, fromage non paraffiné), soit par prélèvement d'une couche de $5 \mathrm{~mm}$ (fromagerie A, fromage paraffiné),

- un échantillon au centre du fromage à l'aide d'une sonde.

\section{Examen microbiologique du lait et du fromage}

Une partie aliquote $(10 \mathrm{~g})$ des échantillons de caillé et de fromage est broyée dans un mortier avec $90 \mathrm{ml}$ d'une solution stérile de citrate de sodium à 2 p. 100 préalablement chauffée à $45^{\circ} \mathrm{C}$. On effectue ensuite des dilutions dans une solution de Ringer au 1/4.

Les échantillons de lait sont dilués au 1/10 dans la solution de Ringer au 1/4. Par ensemencement en boîte de Petri, on a dénombré comme indiqué ci-dessus les groupes microbiens suivants :

- germes totaux sur gélose nutritive [4],

- streptocoques sur gélose nutritive additionnée de 0,5 p. 1000 d'acétate de thallium [6],

- lactobacilles sur milieu de Rogosa [17],

- microcoques et staphylocoques sur milieu de Chapman [2],

- levures et moisissures sur milieu OGGA [9], 
TABLEAU 1. - Stades de la fabrication

I. Lait de brebis pasteurisé + levain + présure.

II. Coagulation à $30^{\circ} \mathrm{C}-32^{\circ} \mathrm{C}$ en $30 \mathrm{mn}$.

III. Découpage du caillé et formation de grains de la dimension d'un petit pois.

IV. $\left\{\begin{array}{l}\text { A. - Réchauffage du grain jusqu'à } 40^{\circ} \mathrm{C} \text { (temps em- } \\ \text { B. - Réchauffage du grain jusqu'à } 19^{\circ} \mathrm{C} \text { pendant }\end{array}\right.$ $20 \mathrm{mn}$.

V. Séparation du petit-lait.

VI. Remplissage des moules et pressage pendant $8 \mathrm{~h}$.

VII. Salage dans un bain de saumure à $22^{\circ} \mathrm{B}$ pendant $48 \mathrm{~h}$.

VIII. *A. - Séchage à $12^{\circ} \mathrm{C}$ pendant $2 \mathrm{j}$.

IX. *A. - Paraffinage du fromage.

$\mathrm{X}$. Mise en cave à $12^{\circ} \mathrm{C}, 85 \mathrm{p} .100$ d'humidité pour affinage:

$1^{\mathrm{e}}$ semaine

$2^{\mathrm{e}}$ semaine

$3^{\text {e }}$ semaine

$4^{\text {e }}$ semaine

$5^{e}$ semaine

$6^{\mathrm{e}}$ semaine

$7^{\text {e }}$ semaine

$8^{\mathrm{e}}$ semaine

$9^{\text {e }}$ semaine

$10^{e}$ semaine

$11^{\mathrm{e}}$ semaine

$3^{\mathrm{e}}$ mois

$4^{e}$ mois

$6^{\mathrm{e}}$ mois

Prélèvement des

échantillons

\begin{tabular}{c|c}
\hline $\begin{array}{c}\text { froma- } \\
\text { gerie } \\
\text { A }\end{array}$ & $\begin{array}{c}\text { froma- } \\
\text { gerie } \\
\text { B }\end{array}$ \\
\hline &
\end{tabular}

* Les opérations $n^{\circ s}$ VIII et IX ont été effectuées seulement dans la fromagerie A. Dans la fromagerie $\mathrm{B}$ on a mis les fromages en cave après le salage.

- bactéries coliformes sur gélose au désochycholate de sodium (désoxycholate Agar Difco no 420).

\section{Analyses chimiques}

Les mesures et analyses suivantes ont été effectuées sur des parties aliquotes des échantillons de fromage ayant servi à l'analyse bactériologique : 
- mesure électrométrique du $\mathrm{pH}$ avec une électrode de verre [8],

- détermination de la matière sèche par la méthode recommandée par la F.I.L. [10],

- détermination de la teneur en lactose [11],

- détermination de la teneur en acide lactique [15],

- détermination de la teneur en chlorures [12],

- détermination de l'azote total par la méthode Kjeldahl, comme décrit par la F.I.L. [13],

- détermination de l'azote soluble après mise en solution du fromage dans l'eau à $50^{\circ} \mathrm{C}[1]$,

- détermination de l'indice d'acide de la matière grasse [14].

\section{RESULTATS}

\section{Evolution de la flore microbienne}

Les résultats de l'analyse microbiologique du lait cru de brebis, destiné à la fabrication des fromages ont donné un fort ensemencement dans les deux fromageries étudiées : $1,1 \times 10^{8}$ germes totaux par $\mathrm{ml}$ pour la fromagerie $\mathrm{A}$ et $2,1 \times 10^{t}$ pour la fromagerie $\mathrm{B}$; en plus des bactéries lactiques (streptocoques et lactobacilles) la teneur du lait en bactéries coliformes est très importante surtout dans l'échantillon $\mathrm{A}$ où la flore coliforme atteint un taux de $10^{\circ}$ germes $/ \mathrm{ml}$.

Après pasteurisation, le nombre de germes totaux a diminué considérablement : $10^{5}-10^{6}$ germes $/ \mathrm{ml}$ pour les deux fromageries, et les germes coliformes ont disparu. La pasteurisation du lait semble donc être plus que justifiée.

Les figures 1, 2, 3 et 4 montrent les variations de la flore microbienne totale et les principaux groupes de bactéries, en surface et au centre des fromages, au cours des différents stades de la fabrication et de l'aftinage. Pour la fromagerie $\mathbf{A}$, où les fromages sont paraffinés après salage et sèchage, l'évolution de la flore est sensiblement identique au centre (fig. 1) et à la surface (fig. 2) du fromage, sauf pour les bactéries halophiles (microcoques et staphylocoques).

Le nombre de germes totaux, très important après l'addition du levain (voisin de $10^{10} / \mathrm{g}$ ), diminue d'une façon remarquable pendant la première semaine après l'emprésurage, avant de se stabiliser.

Le dénombrement des streptocoques indique qu'au delà du $3^{\text {e }}$ jour et durant le $1^{\text {er }}$ mois, ces organismes sont très largement dominants et leur nombre est sensiblement égal à celui des germes totaux, principalement au centre du fromage.

La diminution importante du nombre de germes totaux pendant les 3 premiers jours pourrait être expliquée si l'on tient compte des 


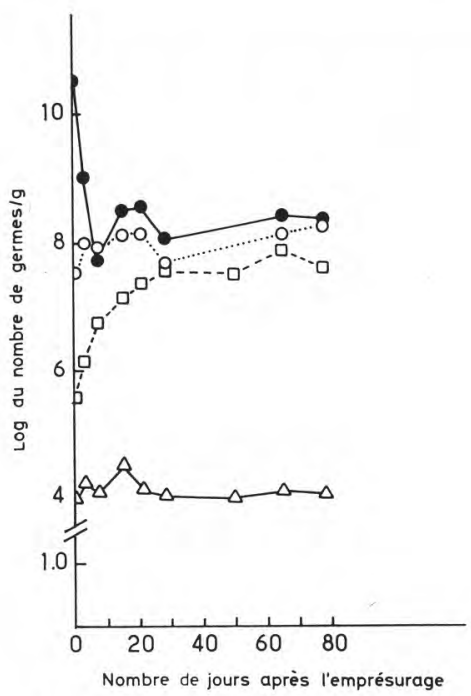

Fig. 1 : Evolution de la flore microbienne au centre des échantillons de fromage "Manchego " (fromagerie A): - - flore totale ; 0 - streptocoques ; 口- lactobacillus; $\Delta$ - microcoques.

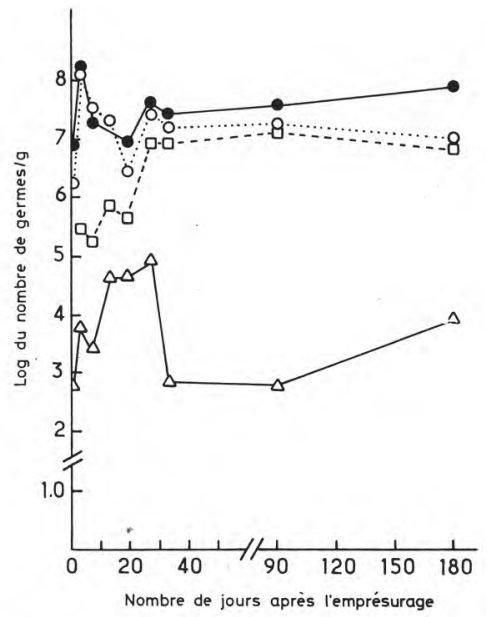

Fig. 3 : Evolution de la flore microbienne au centre des échantillons de fromage "Manchego "(fromagerie B) : - - flore totale ; 0 - streptocoques ; ㅁ - lactobacillus ; $\Delta$ - microcoques.

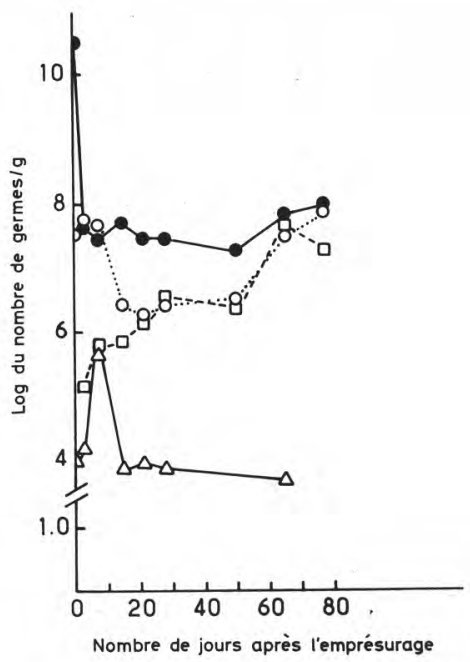

Fig. 2 : Evolution de la flore microbienne à la surface des échantillons de fromage " Manchego » (fromagerie A) : - - flore totale ; 0 - streptocoques ; ㅁ - lactobacillus ; $\triangle$ - microcoques.

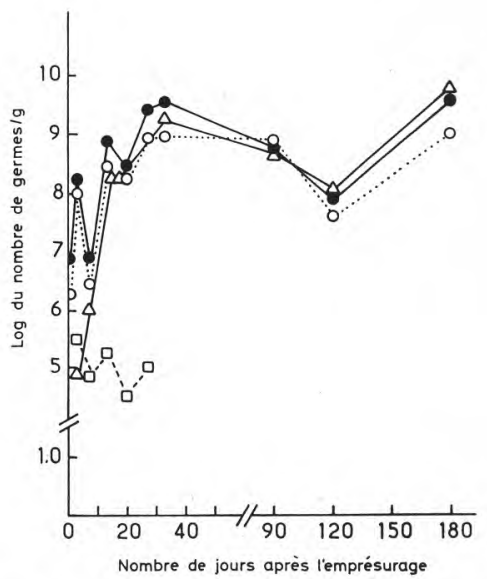

Fig. 4 : Evolution de la flore microbienne à la surface des échantillons de fromage "Manchego »(fromagerie B): - - flore totale ; 0 - streptocoques ; ㅁ - lactobacillus ; $\triangle$ - microcoques. 
résultats des analyses bactériologiques du lait après pasteurisation. En effet, la proportion de streptocoques et de lactobacilles, sur les 10 germes $/ \mathrm{ml}$ dénombrés, était très faible. On peut donc penser qu'une grande partie de la population bactérienne présente dans le lait pasteurisé est détruite par la très forte diminution de $\mathrm{pH}$ (fig. $5 \mathrm{~A}$ ) qui accompagne l'addition du levain et de la présure.

Au cours de l'affinage, tandis que le nombre de streptocoques au centre des fromages reste sensiblement constant (aux environs de $10^{8} / \mathrm{ml}$ ), le nombre de lactobacilles augmente très nettement. Deux mois après l'emprésurage, ces organismes sont présents en quantité aussi importante que les streptocoques. Compte tenu de l'absence de lactobacilles dans le lait après pasteurisation, ainsi que dans le levain, il faut conclure que ces bactéries proviennent de la flore ambiante existant dans la fromagerie. A la surface du fromage, les streptocoques diminuent considérablement à partir du $10^{\circ}$ jour après l'emprésurage pour augmenter ensuite et atteindre de nouveau à la fin de l'affinage la valeur initiale.

Les bactéries halophiles (staphylocoques et microcoques) sont quantitativement fort peu importantes et restent, au centre du fromage, à peu près constantes au cours de la maturation.

A la surface de ces fromages les bactéries halophiles augmentent rapidement en nombre après le salage pour revenir ensuite à leur taux initial.

Les résultats des dénombrements bactériologiques pour la fromagerie B sont montrés dans les figures 3 et 4 . Dans cette laiterie, où les fromages ne sont pas paraffinés, les différences entre les résultats obtenus pour les échantillons de la surface (fig. 4) et du centre (fig. 3) du fromage, sont très importantes.

A l'intérieur, la flore totale augmente de $9 \times 10^{6}$ à $2 \times 10^{\circ}$ germes $/ \mathrm{ml}$ pendant les 3 premiers jours après l'emprésurage et diminue ensuite pour rester sensiblement constante aux environs de $0,5 \times 10^{z}$ germes $/ \mathrm{ml}$ jusqu'à la fin de l'affinage. Les streptocoques suivent les variations de la flore totale et constituent le groupe microbien dominant tout au long de la maturation. Il faut noter cependant que les lactobacilles, présents en petit nombre au début de la maturation, augmentent très nettement durant le $1^{\text {er }}$ mois pour devenir ensuite aussi importants que les streptocoques.

Les microcoques et staphylocoques, partie négligeable de la flore du caillé, augmentent d'une façon importante après le salage et jusqu'au $1^{\text {er }}$ mois, avant de retomber aux faibles concentrations de départ $\left(0,5 \times 10^{3}\right.$ germes $\left./ \mathrm{ml}\right)$.

A la surface du fromage, les streptocoques suivent aussi les variations de la flore totale, mais ces variations en "dents de scie » (fig. 4) ne sont peut-être pas significatives : elles pourraient être dues à l'hétérogénéité des échantillons qui correspondent à différents 
fromages. Il est possible, en effet, que dans un même lot, il existe des différences d'un fromage à l'autre.

A partir des 10 premiers jours, les bactéries halophiles représentent une partie essentielle de la flore superficielle de ces fromages: leur nombre est équivalent au nombre de streptocoques.

L'évolution des lactobacilles à la surface des fromages de la laiterie B n'a pû être correctement suivie à cause du fort peuplement en levures de ces échantillons $\left(10^{7}\right.$ à $10^{8}$ levures/g fromage $)$.

En effet, nous avons constaté que le milieu Rogosa utilisé pour le dénombrement des lactobacilles n'est pas sélectif à 100 p. 100 . La plupart des colonies (provenant des échantillons de surface), qui s'étaient développées sur ce milieu, étaient des levures. Il est fort probable que l'énorme quantité de levures présentes dans ces échantillons inhibe la croissance des lactobacilles. Pour les fromages de la laiterie A, les levures et les champignons ne sont présents qu'en nombre très faible (quelques dizaines de milliers de germes par gramme) du début à la fin de l'affinage.

Nous n'avons pas décelé de bactéries coliformes dans les échantillons correspondant aux différentes étapes de la maturation des fromages de la laiterie B. Par contre, nous avons trouvé un taux de bactéries coliformes, oscillant entre $10^{4}$ et $10^{6}$ germes $/ g$, pour les fromages de la laiterie A. Les analyses effectuées nous permettent de conclure que ces organismes proviennent de la forte contamination des installations dans cette fromagerie.

\section{Variation de la composition chimique des fromages au cours de la maturation}

Pour pouvoir mieux comprendre l'évolution de la flore microbienne au cours de la maturation, différentes analyses chimiques ont été effectuées sur les échantillons de fromage, parallèlement aux analyses bactériologiques.

Les figures $5 \mathrm{~A}$ et $5 \mathrm{~B}$ montrent les variations du $\mathrm{pH}$ et de la teneur en acide lactique au cours de l'affinage des fromages.

Pour les fromages de la série $\mathrm{A}$, on constate que le $\mathrm{pH}$ diminue brusquement après l'addition du levain et de la présure, et se maintient ensuite pratiquement constant. La teneur en acide lactique suit une variation opposée à celle du $\mathrm{pH}$. En effet, nous observons une augmentation de la teneur en acide lactique coïncidant avec la diminution brusque de $\mathrm{pH}$. Par la suite, elle diminue et reste sensiblement constante, jusqu'à la fin de l'affinage, à un taux voisin de $4 \mathrm{mg} / \mathrm{g}$. On peut voir sur la figure $5 \mathrm{~A}$ que pour ces fromages paraffinés, les valeurs du $\mathrm{pH}$ et de la teneur en acide lactique pour les échantillons de surface du fromage ne sont pas significativement différentes de celles obtenues pour les échantillons du centre. Par contre, pour les fromages provenant de la laiterie B (fig. 5 B) nous 


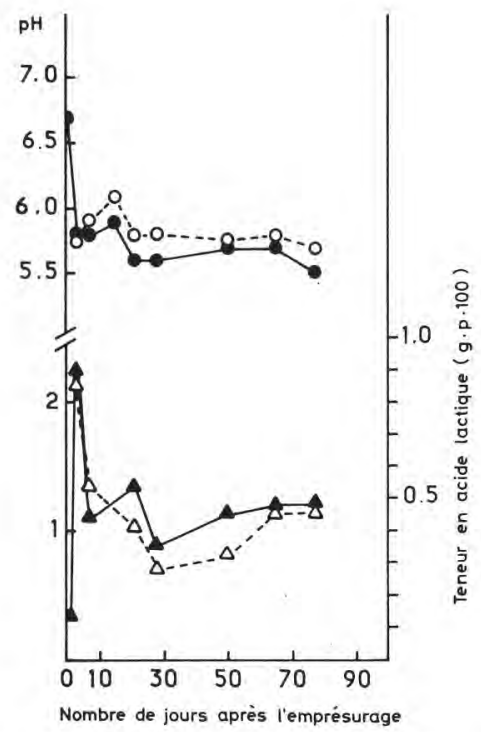

Fig. $5 \mathrm{~A}$ : Teneurs en acide lactique et valeurs de $\mathrm{pH}$ à la surface et au centre des échantillons de la fromagerie A : - intérieur ; - surface ; $\bullet \mathrm{pH}$; $\Delta \Delta$ acide lactique.

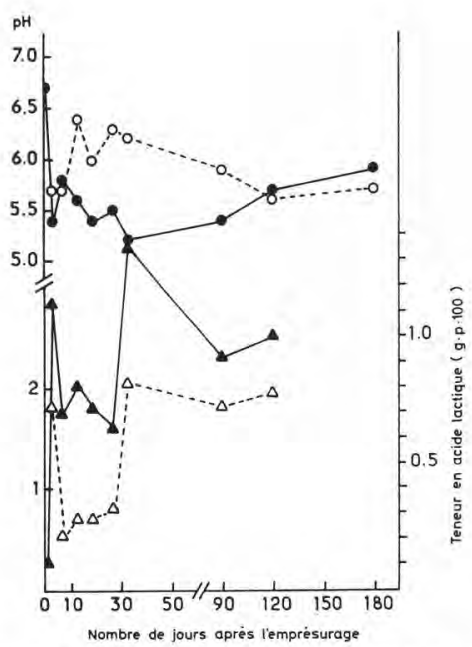

Fig. $5 B$ : Teneurs en acide lactique et valeurs de $\mathrm{pH}$ à la surface et au centre des échantillons de la fromagerie B : - intérieur ; - surface ; $\bullet \mathrm{pH}$; $\Delta \Delta$ acide lactique.

observons d'importantes différences quantitatives pour ces valeurs entre le centre et la surface du fromage.

Quantitativement, ces résultats sont très similaires à ceux observés pour la fromagerie A. Cependant, nous avons constaté, pour les fromages de la série $B$, une deuxième augmentation de la teneur en acide lactique 4 semaines après l'emprésurage.

Nous ne pouvons donner une explication claire pour ce phénomène mais on serait tenté d'attribuer cette augmentation anormale à l'hétérogénéité des échantillons provenant de différents fromages au cours de la maturation.

Les variations de la teneur en chlorure de sodium et en matière sèche sont représentées dans la figure $6 \mathrm{~A}$ pour les échantillons de la fromagerie $\mathrm{A}$, et dans la figure $6 \mathrm{~B}$ pour ceux de la fromagerie $\mathrm{B}$.

Dans les deux cas, on observe que la teneur en chlorure de sodium est très importante à la surface du fromage au moment du salage. Après le salage, on constate une diminution progressive de la teneur en chlorure de sodium de la partie superficielle, salée, qui est accompagnée d'une augmentation de la teneur en sel au cour du fromage. Cette augmentation est plus marquée pour les échan- 


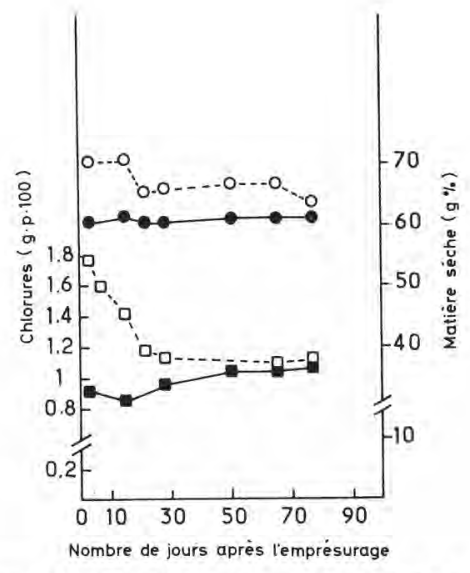

Fig. 6 A: Teneurs en matière sèche et en sel (chlorures) à la surface et au centre des échantillons de la fromagerie A : - intérieur ; . surface ; $\mathbf{= \square}$ chlorures ; 0 matière sèche.

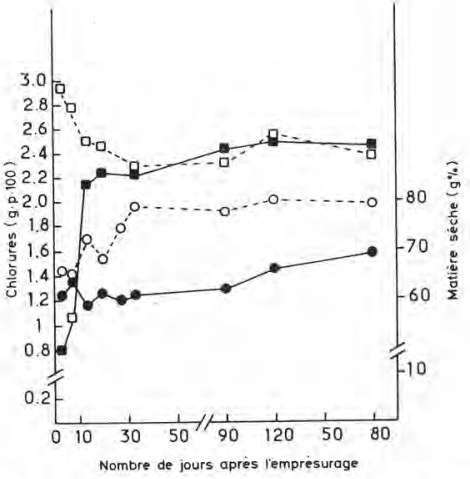

Fig. $6 B$ : Teneurs en matière sèche et en sels (chlorures) à la surface et au centre des échantillons de la fromagerie $\mathbf{B}$ : - intérieur ; .. surface ; $\mathbf{\text { a }}$ chlorures ; 0 matière sèche.

tillons de la fromagerie B, dont la teneur en sel dans la surface au moment du salage est également supérieure à celle des échantillons de la fromagerie A.

La matière sèche reste sensiblement constante au cours de l'affinage pour les échantillons du centre des fromages provenant des deux fromageries. Les échantillons de la surface montrent, par contre, une légère diminution de la matière sèche au cours de la maturation pour la fromagerie $\mathbf{A}$ et une sensible augmentation pour la fromagerie $\mathrm{B}$.

Les résultats des analyses de l'azote total et de l'azote soluble des différents échantillons au cours de l'affinage, donnant une indication de l'évolution de la protéolyse, sont représentés dans les figure $7 \mathrm{~A}$ et $7 \mathrm{~B}$, où nous avons également figuré la variation de l'indice d'acide de la matière grasse. Pour la fromagerie A, le pourcentage d'azote soluble augmente graduellement au cours de la maturation (de 10 à $15 \mathrm{p} .100$ pour l'intérieur, et de 10 à $12 \mathrm{p} .100$ pour la surface), ce qui indique une légère protéolyse au cours de ce processus. Une légère augmentation de l'indice d'acide de la matière grasse est également observée pour ces échantillons, représentant une lipolyse assez faible au cours de la maturation. Dans le cas de la fromagerie B, la protéolyse semble être plus importante que pour la fromagerie A, aussi bien au centre qu'en surface. La lipolyse présente une évolution positive pour les échantillons de la surface mais négative pour ceux du centre. 


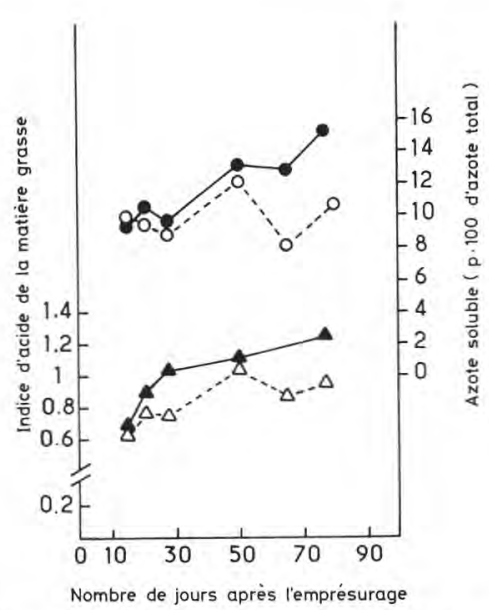

Fig. $7 \mathrm{~A}$ : Variations de la teneur en azote soluble et de l'indice d'acide de la matière grasse à la surface et au centre des échantillons de la fromagerie A : - intérieur ; .. surface ; $\bullet$ azote soluble ; $\Delta \triangle$ indice d'acide.

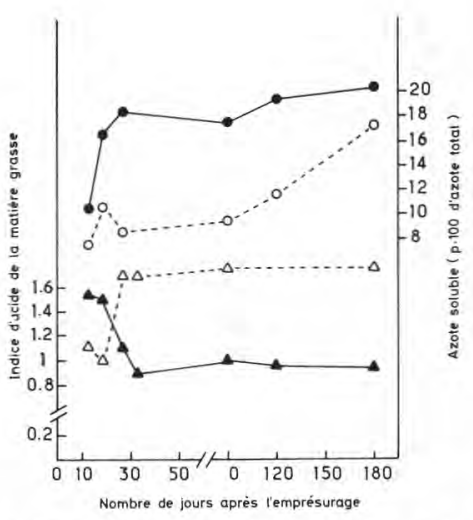

Fig. $7 \mathrm{~B}$ : Variations de la teneur en azote soluble et de l'indice d'acide de la matière grasse à la surface et au centre des échantillons de la fromagerie B: - intérieur ; - - surface ; azote soluble : $\Delta \Delta$ indice d'acide.

\section{DISCUSSION}

Malgré les différences notables trouvées dans l'analyse microbiologique et chimique des fromages élaborés dans les deux fromageries sur lesquelles a porté cette étude, une conclusion nette peut être tirée en ce qui concerne le type de micro-organismes jouant un rôle dominant dans l'affinage du fromage " Manchego ».

Nos résultats ont montré que les streptocoques se trouvent toujours en flore dominante au cours de la maturation tant au centre qu'à la surface des deux séries de fromages analysés. L'analyse bactériologique du levain utilisé dans les deux laiteries permet de conclure que cette flore streptococcique provient en partie des levains ajoutés au lait pasteurisé. Ces streptocoques seraient responsables de la transformation du lactose du caillé en acide lactique, phénomène observé les premiers jours avec une diminution importante du $\mathrm{pH}$. Cette diminution de $\mathrm{pH}$ permet par la suite le développement des lactobacilles dont le nombre augmente au cours de l'affinage pour arriver, au-delà du $1^{\text {er }}$ mois, à une concentration aussi importante que celle des streptocoques. L'évolution positive de la flore lactobacillaire au cours de la maturation et son importance numérique dans les dernières étapes de la maturation du fromage suggèrent que les lactobacilles ont un rôle non négligeable dans le développement du goût caractéristique du fromage " Manchego ». 
Les résultats des études concernant les souches de lactobacilles et qui feront l'objet d'un prochain rapport, nous ont permis de constater que la majeure partie de ces souches, isolées sur milieu de Rogosa à partir des échantillons prélevés à différents stades de la maturation, appartient aux espèces $L$. plantarum et $L$. casei.

Les microcoques et les levures sont relativement peu nombreux tout au long de la maturation à l'intérieur des fromages provenant des deux fromageries étudiées. Bien que, après le salage, l'on observe une augmentation considérable de la population de microcoques et levures dans la surface des fromages non paraffinés (provenant de la fromagerie B), nous croyons que cette population ne joue aucun rôle dans le processus de maturation de ces fromages.

Il est intéressant de noter que dans d'autres types de fromages comme le Saint-Paulin [4] ou le Roquefort [3] les lactobacilles ne représentent qu'une faible partie de la microflore totale. Le fort développement de la flore lactobacillaire, observé surtout dans les dernières étapes de l'affinage du fromage "Manchego », fait penser qu'une expérimentation systématique portant sur l'inclusion de différentes souches de lactobacilles dans le levain utilisé pour la fabrication de ce type de fromage devait donner des orientations intéressantes pour une amélioration du processus de fabrication du « Manchego ».

\section{Remerciements}

Nous remercions Mme Isabel Martinez Castro pour son aide dans la réalisation des analyses chimiques.

\section{R és u m é}

L'évolution de la flore microbienne et des caractéristiques chimiques au cours de la fabrication et de l'affinage du fromage espagnol " Manchego » ont été étudiées sur des échantillons prélevés dans deux fromageries de la région de Madrid. Les germes totaux, les streptocoques, les microcoques et staphylocoques, les lactobacilles, les levures et moisissures, et les bactéries coliformes ont été dénombrés.

Nous avons observé que les streptocoques constituent la flore dominante de ces fromages à tous les stades de l'affinage. Les microcoques et les levures restent relativement peu nombreux pour les échantillons provenant des fromages paraffinés (laiterie A) et pour l'intérieur des fromages non paraffinés (laiterie B). Par contre, les microcoques ainsi que les levures constituent une partie très importante de la flore de la surface des fromages de la laiterie B. Quant 
aux lactobacilles, qui proviennent surtout de la microflore ambiante, ils se développent au cours de l'affinage et forment à la fin de la maturation une partie notable de la microflore totale, aussi importante que les streptocoques.

\section{S u m mary}

A study of the microbial flora and chemical characteristics of the "Manchego » cheese has been carried out during the different stages of making and ripening of two batches of cheeses coming from two different factories (A and B). The microbial groups analysed included the streptococci, the staphylococci, the micrococci, the yeast and molds, the lactobacilli and the coliform organisms.

The streptococci were found to be the predominant organisms throughout the ripening process in both lots of cheeses. The micrococci, yeasts and molds were present in only small amounts in the samples from the surface and the center of the paraffined cheeses (coming from factory A) and in the samples from the center of the non-paraffined cheeses (factory B). On the contrary, the micrococci were among the main organisms to be found on the surface of the cheeses coming from factory B. Finally the lactobacilli developped during the process of ripening and in the late stages they were found to be numerically as important as the streptococci.

Reçu pour publication le 23 décembre 1974.

\section{Références bibliographiques}

[1] Casares (R.) (1967). - Tratado de Análisis Químico. Tomo III. Editorial Casares, Madrid.

[2] Chapman (G. H.) (1945). - The significance of sodium chloride on studies of staphylococci. J. Bact., 50, 201.

[3] Devoyod (J. J.) (1970). - La flore microbienne du fromage de Roquefort. V. Les lactobacilles. Le Lait, 495-496, 1-8.

[4] Ducastelle (A.), Lenoir (J.) (1965). - Contribution à l'étude de la flore microbienne du fromage de type Saint-Paulin. I. Son évolution au cours de la maturation. Le Lait, 447, 371-378.

[5] Harper (W. J.), Kristoffersen (T.) (1956), - Biochemical aspects of cheese ripening. J. Dairy Sci., 39, 1773-1775.

[6] Hirsch (A.), Mc Clintock (M.), MocQuot (G.) (1952). - Observations on the influence of inhibitory substances produced by the lactobacilli of Gruyère cheese on the development of anaerobie sporeformers. J. Dairy Res., 19, 179-186.

[7] Ministerio de Agricultura (1969). - Catálogo de Quesos Espanoles. Direccíon General de Ganadería, Madrid.

[8] Ministère DE L'Agriculture (1969). - Contrôle de la qualité des produits laitiers. Tome I, Analyses Physique et Chimique VII-9. 
[9] Mossel (D. A. A.), Visser (M.), Mengenin (1962). - A comparison of media used for the enumeration of moulds and yeasts in foods and beverages. Lab. Pract., 11, 109-112.

[10] Norme Internationale. F.I.L.-I.D.F., 4, 1958.

[11] Norme Internationale. F.I.L.-I.D.F., 43, 1967.

[12] Norme Internationale. F.I.L.-I.D.F., 17, 1961.

[13] Norme Internationale. F.I.L.-I.D.F., 25, 1964.

[14] Norme Internationale. F.I.L.-I.D.F., 6 A, 1969.

[15] Official Methods of A.O.A.C. (1970) n $\mathrm{n}^{\circ} 16201$.

[16] Orla Jensen (S.) (1940). - Théorie de la maturation des fromages durs. Le Lait, 20, 2-15.

[17] Rogosa (M.), Mitchell (J. A.), Wiseman (R. F.) (1951). - A selective medium for the isolation and enumeration of oral and fechal lactobacilli. J. Bact., $62,132$. 